\title{
Fine structure of Drosophila larval salivary gland ducts as revealed by laser confocal microscopy and SEM
}

\author{
Denisa BEŇOVÁ-LISZEKovÁ (D), MILAN BEŇO and Robert FARKAŠ* (D)
}

Laboratory of Developmental Genetics, Institute of Experimental Endocrinology, Biomedical Research Center, Slovak Academy of Sciences, Dúbravská cesta 9, 84505 Bratislava, Slovakia; e-mails: denisa.benova-liszekova@savba.sk, milan.beno@savba.sk, ueenfark@savba.sk

Key words. Diptera, Drosophilidae, Drosophila, larval salivary glands, secretory glue, salivary duct, taenidia, ultrastructure

\begin{abstract}
The functions of the larval salivary glands (SGs) of Drosophila are traditionally associated with the production of a massive secretion during puparium formation; it is exocytosed into a centrally located lumen and subsequently expectorated via ducts, the pharynx and mouth. This so-called proteinaceous glue serves as an adhesive to attach the puparial case to a solid substrate. Great attention has been paid to the secretory cells of SGs, which are famous for their giant polytene chromosomes. However, substantially less attention has been devoted to individual or common ducts that form the most proximal portion of the SG organ via which the glue is released into the pharynx. In the present paper, we describe the organization and fine structure of the taenidia, highly specialized circumferential ring-like extracellular (cuticular) components on the internal side of these tubes. Two chitin-specific probes that have previously been used to recognize taenidia in Drosophila tracheae, Calcofluor White M2R (also known as Fluorescent Brightener 28) and the novel vital fluorescent dye SiR- $\mathrm{COOH}$, show positively stained ductal taenidia in late larval SGs. As seen using scanning electron microscopy (SEM), the interior of the ductal tube contains regular and denselyarranged ridge-like circumferential rings which represent local thickenings of the cuticle in various geometries. The microtubular arrays that optically colocalize with taenidia in both the trachea and SG ducts are specifically and strongly recognized by fluorescently-conjugated colchicine as well as anti-tubulin antibody. In contrast to taenidia in the tracheae, the analogous structures in SG ducts cannot be detected by fluorescently-labeled phalloidin or even actin-GFP fusion protein, suggesting that the ducts lack a cortical network made of filamentous actin. We speculate that these taenidia may serve to reinforce the duct during the secretory processes that SGs undergo during late larval and late prepupal stages.
\end{abstract}

\section{INTRODUCTION}

The larval salivary glands (SGs) of Drosophila melanogaster represent a unique model organ. They are best known for their giant polytene chromosomes, the detailed analysis of which led to several important advances, including establishment of the first cytogenetic maps and the elaboration of chromomere theory (Painter, 1933; Bridges, 1935; Pelling, 1966; Ashburner, 1970; Lefevre, 1976; Sorsa, 1989; Lindsley \& Zimm, 1992). Furthermore, the degree of polyteny of SG chromosomes has made it possible to study the puffs which represent active gene sites. The main function of the larval SG appears to be the production of a mucinous glue, which serves as an adhesive during puparium formation (Fraenkel \& Brookes, 1953). In anticipation of this function, at about the middle of the third-instar, globular, highly refractile PAS-positive granules appear in the cytoplasm of SG cells (Berendes, 1965; Kolesnikov \& Zhimulev, 1975). The hormone ecdysone plays a dual role in this process, by both starting and then terminating a specific developmental program of protein synthesis required for the production of these secretory granules. Upon a dramatic increase in the ecdysone titer a few hours prior to pupariation, the contents of the granules are released by exocytosis into the SG lumen, which is then emptied by expectoration during puparium formation (Lane et al., 1972; von Gaudecker, 1972; Berendes \& Ashburner, 1978; Farkaš \& Šut'áková, 1998) where it serves as a glue to attach the puparial case to a solid substrate (e.g. vial glass wall). Thus, these granules constitute the components of the salivary glue secretion (Sgs). The Sgs represents a highly specialized and unique extracellular composite bioadhesive secretion, the individual protein components of which are encoded by a series of eight Sgs genes (Korge, 1975, 1977a, b; Ashburner \& Berendes, 1978; Lehmann, 1996; Farkaš, 2016) that are known to form a set of so-called interecdysial chromosomal puffs.

\footnotetext{
*Corresponding author; e-mail: ueenfark@savba.sk
} 
Individual $S g s$-genes at these cytological bands have been subsequently cloned and molecularly characterized (reviewed in Farkaš, 2016; Da Lage et al., 2019).

In the course of our SEM studies on the surface properties and internal substructure of exocytosed and expectorated glue (Beňová-Liszeková et al., 2019b), we found that the internal lining of the tubular, non-secretory structure of the SG, i.e., the duct that develops during embryogenesis by the coordinated action of a differently expressed set of genes, in contrast to the SG proper (Kuo et al., 1996; Jones et al., 1998; Yao \& Sun, 2005), shows peculiar structures resembling tracheal taenidia. Using classical light microscopy, the potential presence of taenidial-like chitinous circumferential rings in Drosophila SG duct was noticed by Robertson (1936) and Ross (1939). Their work was cited by Bodenstein (1950) and by Berendes \& Ashburner (1978). Then, there was an informational gap on taenidia until Haberman et al. (2003) studied the embryonic specification of cell fates within the salivary gland primordium, including salivary ducts, where they observed the formation of taenidialike structures during the embryogenesis of $D$. melanogaster; however, no additional attention was paid to the SG ducts. The taenidia are distinctive to the insect tracheal system, and are defined as circumferential thickenings of the cuticle inside a trachea or tracheole where they show various geometries such as loops or spirals (Snodgrass, 1935; Richards, 1951; Eidmann \& Kühlhorn, 1970; Wigglesworth, 1972; Mill, 1972, 1998; Noirot \& Noirot-Timothee, 1982; Manning \& Krasnow, 1993). Using laser confocal microscopy and SEM we show herein that the larval SGs of Drosophila contain microtubule-lined taenidial structures inside the ductal tubes, similar to those present in the tracheae, but in which filamentous actin is conspicuously absent.

\section{MATERIALS AND METHODS}

\section{Abbreviations}

FITC - fluorescein isothiocyanate; GFP - green fluorescent protein; HMDS - hexamethyldisilazane; PAS - periodic acidSchiff stain; PBS - phosphate-buffered saline; (PBT) - PT + bovine serum albumin; PBTS - PBT + goat serum; PT - PBS + Triton X-100; SEM - scanning electron microscopy; SG - salivary gland; $\mathrm{Sgs}$ - salivary glue secretion; $\mathrm{SiR}$ - silicone rhodamine; TEM - transmission electron microscopy.

\section{Fly culture and staging}

Flies from the Oregon $R$ wild type stock of fruit flies (Drosophila melanogaster, Meigen) were grown in $50 \mathrm{ml}$ vials or 200 $\mathrm{ml}$ bottles at $23^{\circ} \mathrm{C}$ on agar-yeast-cornmeal-molasses medium (Ashburner \& Thompson, 1978; Ransom, 1982) with the addition of methylparaben (Sigma) to prevent molds. For the experiments described herein, SGs of pre-wandering (105-110 h after egg laying) and wandering (116-120 h after egg laying) third-instar larvae were used.

Besides wild type, the following genotypes of flies were used: $P\{w[+m C]=A c t G F P\} J M R 3, P\{A c t G F P . w[-]\} C C 2, U A S p-$ Act5C.T:GFP, $P\{w[+m C]=A c t G F P\} J M R 1$, and $P\{w[+m C]=$ $A c t G F P$ \}JMR1. To control the expression of UAS-target constructs, three types of Gal4 drivers were used: Sgs3-Gal4, driGal4 and $h s$-Gal4. With the exception of Sgs3-Gal4, the gift of
A.J. Andres, all stocks were obtained from Bloomington Drosophila Stock Center.

\section{Immunohistochemistry and confocal microscopy}

Salivary glands were dissected from the larvae in Ringer solution using extrafine Dumont \# 5 tweezers under a Wild M3Z or Leica MZ9,5 stereomicroscope and fixed in PIPES-buffered 4\% paraformaldehyde (20 mM PIPES, $60 \mathrm{mM}$ sucrose, $1 \mathrm{mM}$ EGTA, $5 \mathrm{mM} \mathrm{MgCl}_{2}, \mathrm{pH}$ 7.2). In order to stain tissues with antibodies, the tissues were permeabilized with $0.1 \%$ Triton X-100 in PBS (PT) and then blocked with PT containing $2 \%$ fraction $\mathrm{V}$ of bovine serum albumin (PBT; Serva) and $2 \%$ goat serum (PBTS; Sigma). To detect microtubules, primary rabbit anti- $\beta$-Tubulin polyclonal antibody (Sigma \# T-3526) diluted 1:200 was added, before incubating overnight at $4^{\circ} \mathrm{C}$ in the presence of PBTS. To detect the borders (membranes) of individual ductal cells, primary mouse anti-Fasciclin III monoclonal antibody (DSHB \# 7G10) diluted 1:10 was used. After intense washing in PT, goat affinity purified and preadsorbed Cy5-anti-mouse $\mathrm{F}(\mathrm{ab})_{2}$-specific and Cy3-anti-rabbit $\mathrm{F}(\mathrm{ab})_{2}$-specific secondary antibodies (Jackson ImmunoResearch Labs.) was added before incubating for $2 \mathrm{~h}$ at room temperature $\left(23-25^{\circ} \mathrm{C}\right)$. As an alternative protocol to detect microtubules, FITC-labeled colchicine (Molecular Probes/Invitrogen) was diluted $1: 200$ before incubating for $2 \mathrm{~h}$ at room temperature. After intense washing in PT, the salivary glands were mounted in Dabco-supplemented (Sigma) Elvanol under a Schött high-performance № $1.5 \mathrm{H}$ coverslip and scanned under a Zeiss LSM-510 META laser confocal microscope using a 40× (NA 1.3) oil objective lens. Collected Z-stack images were deconvolved using Zeiss Axiovision 4.8 or SVI Huygens Professional 4.3 software, rendered at maximum intensity, and further processed in Adobe Photoshop.

To detect chitin (a major component of taenidia) in fixed tissue, SGs (or tracheae) were stained with Calcofluor White M2R (also known as Fluorescence Brightener 28, FB28; Sigma, \# F-3543) at pH 9 in $0.2 \mathrm{M}$ Tris buffer containing $0.1 \%$ Triton X-100 (Serva) for $2-3 \mathrm{~h}$ at room temperature. Whenever necessary, samples were also counterstained with Alexa Fluor 488 or Alexa Fluor 546 phalloidin (Molecular Probes/Invitrogen) to detect filamentous actin, as previously described (Farkaš et al., 2015, 2018).

For live cell in vivo imaging of salivary ducts, freshly dissected salivary glands were uploaded for $1 \mathrm{~h}$ in $1 \mu \mathrm{M}$ SiR-COOH or 1 $\mu \mathrm{M} 580 \mathrm{CP}-\mathrm{LTX}$ at room temperature. SiR-COOH and 580CPLTX are novel silicon-rhodamine and carbopyronine-based fluorescent dyes (Lukinavičius et al., 2018). After incubation, the SGs were briefly rinsed in three volumes of Drosophila saline solution, mounted in Drosophila Ringer containing 20\% glycerol under a Schött high-performance № $1.5 \mathrm{H}$ coverslip, sealed with Picodent nontoxic duplicating silicone or nail polish, and immediately viewed under a Zeiss LSM-510 META or Leica TCS SP8 STED-3X laser confocal microscope using 40× (NA 1.3) and HCX-PL-APO 100× (NA 1.4) oil objective lenses, respectively. Collected Z-stack images were deconvolved using Zeiss Axiovision 4.8 or SVI Huygens Professional 4.3 software, rendered at maximum intensity, and further processed in Adobe Photoshop.

Image fusion of widefield light or phase contrast microscopic pictures was performed by focal plane merging of selected Zstacks via edge detection and Fourier analysis in order to obtain a greater depth of field focus in the final blended image.

\section{Light and phase contrast microscopy}

Salivary gland ducts and tracheae were also imaged under Leica DMRE and Leica DM6000B microscopes equipped with Leica DFC320 digital color cameras controlled by Leica LAS 2.6.0 software. Image fusion of widefield light or phase contrast 
microscopic pictures was performed by focal plane merging of selected Z-stacks via edge detection and Fourier analysis in order to obtain a greater depth of field in the final blended image.

\section{Sample processing for SEM}

Whole salivary glands were fixed in $4 \%$ paraformaldehyde (Polysciences) $+2 \%$ glutaraldehyde (Ted Pella) in $0.1 \mathrm{M}$ sodium cacodylate ( $\mathrm{pH} 7.2$; Serva) for $1 \mathrm{~h}$ at $23-25^{\circ} \mathrm{C}$; the samples were placed in inverted caps from $1.5 \mathrm{ml}$ eppendorf tubes and approximately $100 \mu \mathrm{l}$ of fixative was used per 10 pairs of glands to ensure that they were fully covered. The SGs were then rinsed five times (5-10 min per rinse) in 100-200 $\mu 1$ of clean $0.1 \mathrm{M}$ sodium cacodylate $(\mathrm{pH} 7.2)$ at $23-25^{\circ} \mathrm{C}$. Samples were postfixed in $0.5 \%$ osmium tetroxide (EMS) in $\mathrm{ddH}_{2} \mathrm{O}$ for $30 \mathrm{~min}$ at $23-25^{\circ} \mathrm{C}$, and then extensively rinsed in $\mathrm{ddH}_{2} \mathrm{O}$ (minimum 6 times for $10 \mathrm{~min}$ each). SGs were dehydrated in an ascending series of ethanol $(30 \%$, $50 \%, 70 \%, 96 \%$ and $100 \%$; Merck); the dehydration step with $100 \%$ ethanol was repeated twice before applying a $1: 1$ mixture of $100 \%$ ethanol $+100 \%$ acetone (Merck) twice, followed by three changes of $100 \%$ acetone. At this step it was important that sample did not dry completely during the exchanges of absolute ethanol or acetone, or at any subsequent time before critical point drying. Therefore, the addition of hexamethyldisilazane (HMDS; Sigma-Aldrich) in place of Peldri II to facilitate critical point drying (Beňo et al., 2007; Beňová-Liszeková et al., 2019a), was performed carefully in several steps: the first volume of HMDS was applied in the presence of small remnants of acetone; then, after 5-10 min, the HMDS was quickly removed and fresh HMDS added, taking care that the previous HMDS did not dry up; finally, SGs were kept in HMDS for $30 \mathrm{~min}$, and then the remnants of the HDMS were allowed to evaporate completely. The process of HMDS evaporation has a tendency to generate a slight electrostatic potential on the surface of samples, thus the last step was performed in a very clean air environment (e.g. under a desiccator) to minimize the attraction of dust particles.

Dried SGs were mounted on pieces of double-sided tape (Scotch) on 16 or $24 \mathrm{~mm}$ aluminum SEM stubs and then sputtered for 2.5 min with gold-palladium using a Balzers sputter coater device SCD-030 at 35-40 mA per stub and at a pressure of 0.05 to 0.1 mbar. This produced a $40-50 \mathrm{~nm}$ continuous alloy layer. Samples were viewed and photographed using an FEI Quanta FEG250 scanning electron microscope with the emission field cathode at an accelerating voltage of $10 \mathrm{kV}$. The bitmap images obtained were processed and labeled using Adobe Photoshop or Corel Draw software and assembled into figures using Adobe Photoshop.

\section{RESULTS}

\section{Tubular ducts of salivary glands}

The Drosophila larval SG is a paired organ which is split into the SG proper and the tubular duct (Fig. 1). In contrast to the widely studied SG proper, little attention has been paid to duct morphology; to our knowledge its immunohistochemical properties and SEM ultrastructure have not been documented. The tubular duct is the last portion of the salivary gland system through which Sgs-glue must pass to reach the pharynx and mouth, where it is expectorated into the environment. The larval duct is composed of about 55 cuboidal epithelial cells with polytene nuclei that form simple tubes. These tubes connect the secretory cells to the larval mouth by a Y-shaped tubular conduit (Berendes \& Ashburner, 1978; Abrams et al., 2003). The ducts of the

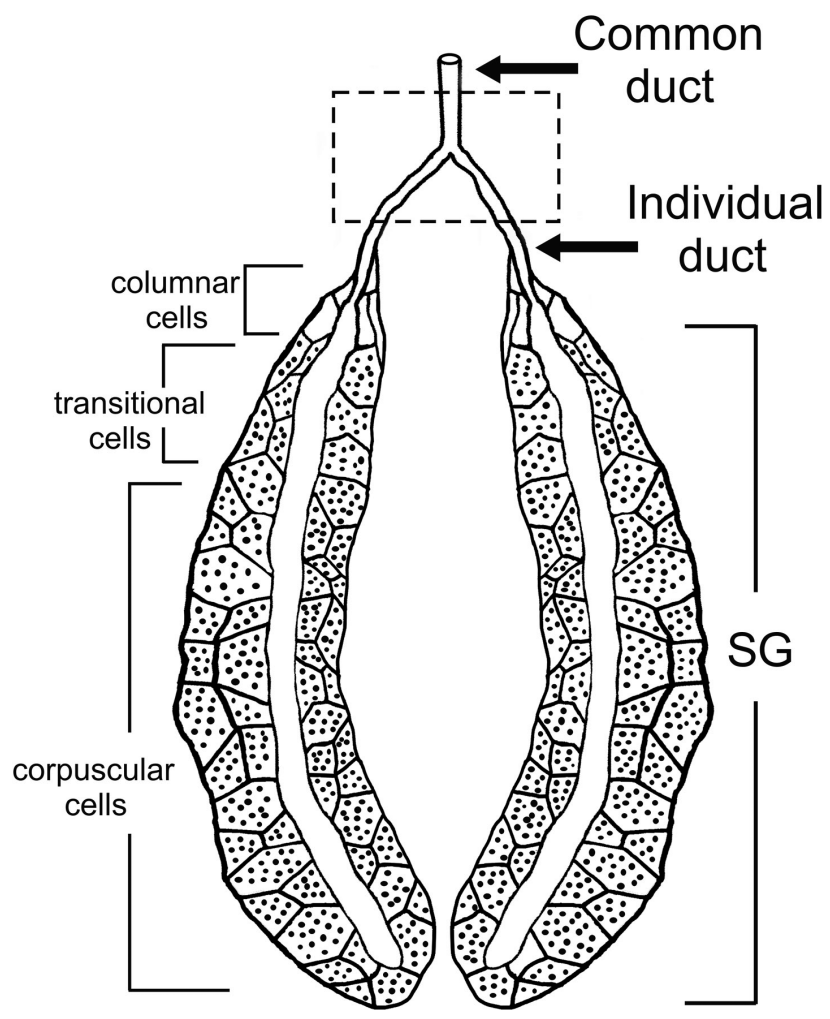

Fig. 1. Schematic view of Drosophila larval salivary glands (SGs) and their ducts (dashed rectangle). Picture represents the SGs of a wandering late third-instar larva, when its secretory cells are filled with Sgs-glue in the form of numerous vesicles (black dots inside SG cells). Arrows point to individual and common ducts of SG. Range of corpuscular, transitional and columnar cells of SG proper is also indicated.

left and right gland unite to form a common duct opening into the floor of the pharynx. This is the only structure of Drosophila SGs which has not been studied in more detail throughout the long history of SG research.

While investigating SGs for another purpose under SEM, we found that the interior of the tubular duct contains regular and densely-arranged ridge-like circumferential rings or taenidia. These represent local thickenings of the cuticle in various geometries such as loops, rings, or helices, as would normally be found in the tracheal system (Fig. 2a-d). The thickness of the individual taenidia in SG ducts is between 280 and $690 \mathrm{~nm}$ (Fig. 2e, f), similar albeit not identical to that of tracheal taenidia (400 to $760 \mathrm{~nm}$ ) from identically aged third-instar larvae, with the distance between taenidia also showing some level of similarity (Fig. 2g, h). However, from these images it is also clear that, unlike in tracheae, taenidia in SG ducts are rough and ladder-like with irregularly-sized randomly distributed side ramifications protruding into the spaces between them. This is in contrast to the smoother surface of tracheal taenidia which have spaces filled with trabecular structure resembling a dense arterial network. Interestingly, on the basal surface of the SG duct (under basal lamina) we observed circular, rope-like surrounding fascicles 250 to $350 \mathrm{~nm}$ in diameter (Fig. 2i,j) which we did not find in tracheae. In this study we were not able to discern their nature and composition. 


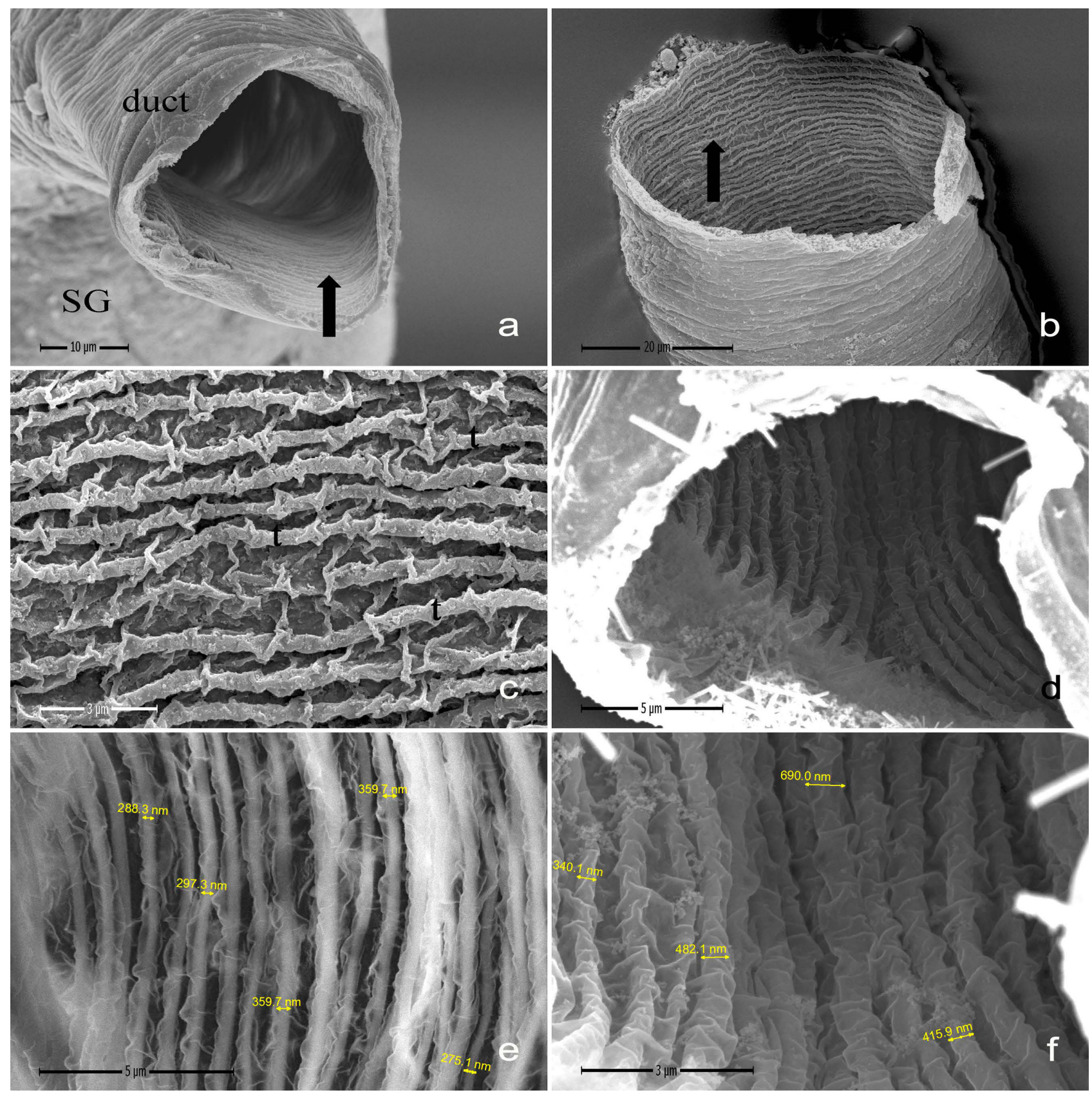

Fig. 2a-f. SEM images of Drosophila salivary gland ducts. (a) Low magnification frontal view of the SG duct interior indicating the presence of densely-arranged ridge-like circumferential rings (black arrow); SG - salivary gland proper. (b) Closer and slightly lateral view of the circumferential arrangement of taenidial rings inside the SG duct (white arrow). (c) Detailed view of ductal taenidia (t) revealing their rough, ladder-like structure with irregularly sized and randomly distributed side ramifications (white arrows) protruding into spaces between them. (d) Taenidia which are closer to the anterior end of the individual duct where they unite to a common Y-shaped tubular conduit appear to be more densely arranged. (e) and (f) Dimensions of these ductal taenidia vary from 280 to $360 \mathrm{~nm}$ for the anterior portion of the duct (e), in contrast to $400-690 \mathrm{~nm}$ for the posterior portion of the duct (f).

\section{Distribution of cytoskeletal and chitin probes in SG ducts}

After initial inspection under wide field light and phase contrast microscopes, which also revealed dense taenidiallike rings inside SG ducts of late third instar larva (Fig. 3a), we tested two chitin-specific probes, that have previously been used to recognize taenidia in Drosophila tracheae, Calcofluor White M2R (also known as Fluostain or Fluorescent Brightener 28) and the novel vital fluorescent dye SiR-COOH (Lukinavičius et al., 2018). Fig. 3b shows positively stained ductal taenidia in late larval SGs of Drosophila using SiR-COOH. The chitinous taenidial character of these structures was further confirmed by their strong/specific fluorescent staining using Calcofluor White M2R (Fig. 3c). In both cases, tracheal tubules stained in parallel with SGs under the same conditions were used as positive controls (Fig. 3d, e). In contrast to tracheae, the intracellular actin lining parallel to taenidia in SG ducts is absent (Fig. 3f), but the chitin signal overlaps strongly with intracellular tubulin recognized by both fluorescentlylabeled colchicine and anti-tubulin antibody (Fig. 3h, i). 

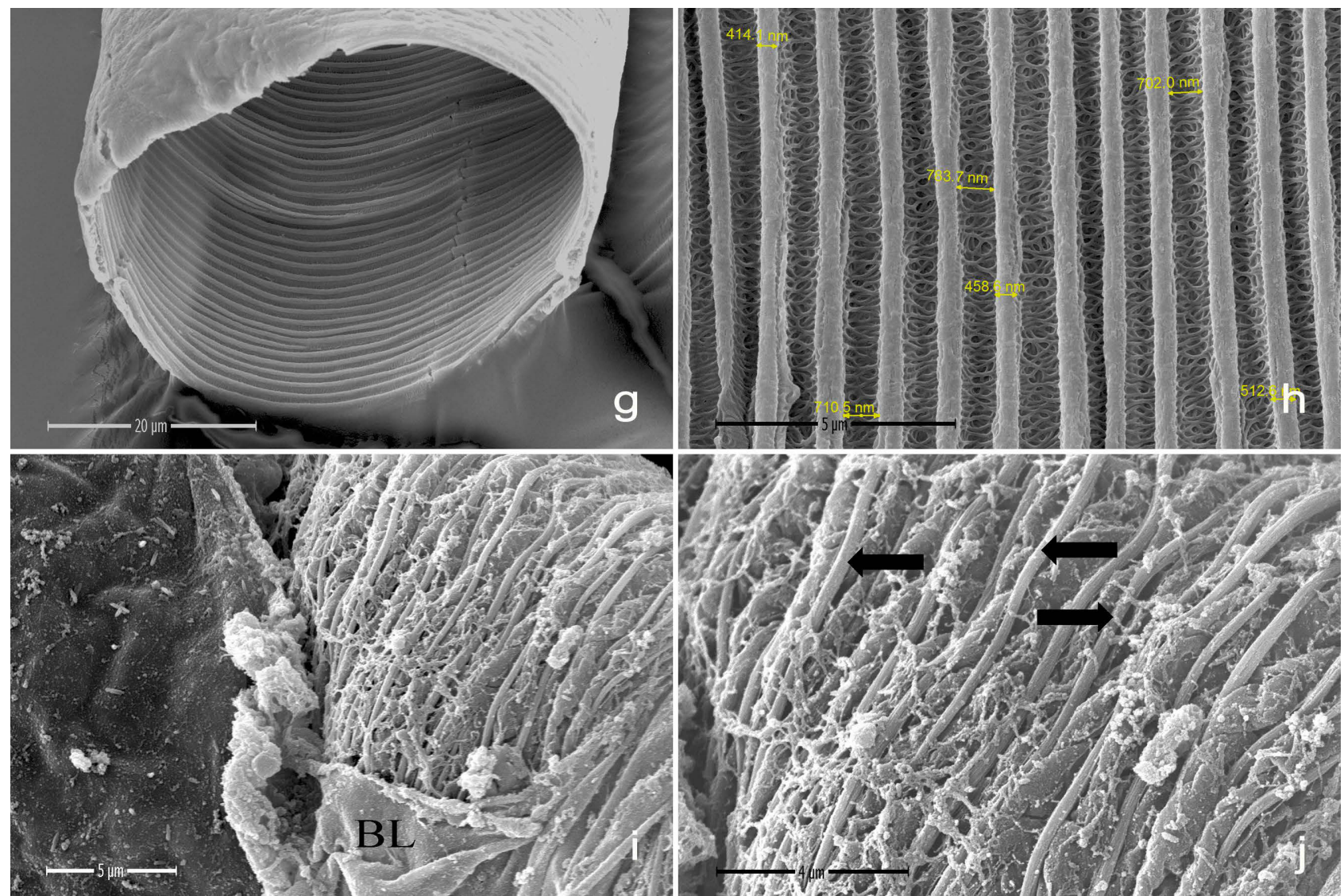

Fig. 2g-j. SEM images of Drosophila tracheae and salivary gland ducts. (g) For comparison of a typical taenidial structure, an overall view of trachea from the same larva as (a) or (b) is shown. (h) Higher magnification view of tracheal taenidia with their more regular and smoother surface, and interspaces filled with trabecular structure resembling a dense arterial network. The space between individual taenidia ranges from 700 to $800 \mathrm{~nm}$. (i) Outside view of a "naked“ SG duct after peeling off a portion of the basal lamina (BL). (j) More detailed view of ropelike circularly surrounding fascicles 250 to $350 \mathrm{~nm}$ in diameter (black arrows).

The distribution of fasciclin-III demarcates the limits of individual ductal cells (Fig. 3g). Identical parallel staining of tracheae (Fig. 3k-n) revealed that, in contrast to SG ducts, tracheal phalloidin-bound F-actin bundles are highly visible (Fig. 3k) and their signal clearly overlaps with the position of microtubules (Fig. 3m) and Fluostain-positive chitin (Fig. 3n). The merge of all four channels (Fig. $3 j$ and 3o) highlights the fact that microtubular and chitinous signals overlap significantly in both tissues. In addition, in Fig. 3o the overlap of microfilamentar, microtubular and chitinous signals in the tracheal trunk is highly apparent. In order to provide a more detailed view of the SG ducts, some of these vital-stained as well as fixed and FB28labelled taenidia are shown at a higher magnification and with a monochrome regime in Fig. 4.

This finding was intriguing, since previous reports described the presence of $\mathrm{F}$-actin bundles parallel to taenidia not only in embryonic SG ducts, but also in ducts of second-instar larvae (Haberman et al., 2003). Their absence in late third-instar larvae was therefore unexpected. To clarify this observation, we decided to verify the binding of the fluorescent probe, phalloidin, to typical locations for filamentous actin such as the cortical cytoskeleton and exocytotic vesicles within the corpuscular secretory cells of SGs. The staining protocol was identical to that of the duct cells, and several observations of both secretory and duct cells were performed from the same microscope slides. As documented in Fig. 5a-d, both Alexa Fluor 488 phalloidin and Alexa Fluor 546 phalloidin bind specifically and strongly to both the cortical cytoskeleton and exocytotic vesicles. Thus, the absence of visible F-actin rings that encircle the entire SG duct was not an artifact or error in the protocol.

\section{Detection of actin-GFP fusion protein in SG ducts}

In the next step we used act-GFP transgenic flies expressing actin-GFP fusion protein ubiquitously in most if not all tissues during development. Out of 5 tested actinGFP fusion lines, $P\{w[+m C]=A c t G F P\} J M R 3$ expresses GFP the most intensely in the SGs of late third-instar larvae and fluorescence could be detected not only in vivo but also after paraformaldehyde fixation. Therefore, this line was used to observe the GFP signal in SG ducts. We found that GFP, either in living SGs or in fixed organs, produces barely visible fluorescence without $\mathrm{F}$-actin rings inside the duct (Fig. 5e, f). This indicates that even the natural expression and distribution of actin-GFP fusion protein does not occupy the positions of the F-actin rings visible in younger stages.

In addition, we also used the UAS-Act5C-GFP line, the expression of which was under Sgs3-Gal4, dri-Gal4 or hsGal4 drivers, to enhance the abundance of Actin-5C-GFP 

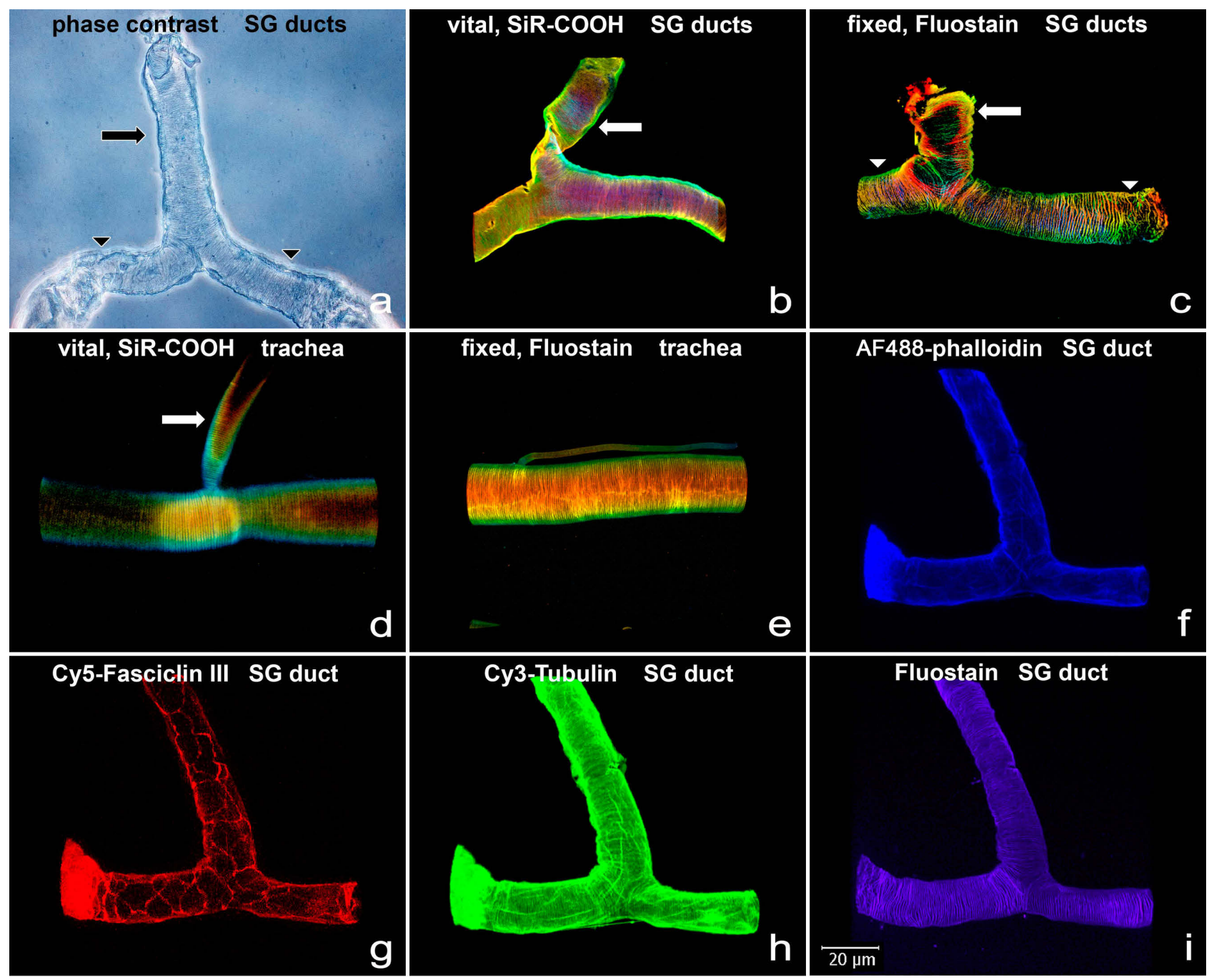

Fig. 3a-i. Salivary gland ducts of Drosophila larvae. (a) Ducts of the left and right gland (black arrowheads) unite to form a common Y-shaped tubular conduit (arrow) directing into the floor of the pharynx. Densely arranged taenidial rings inside SG ducts are evident if viewed under a phase contrast microscope (magnif. 40×). (b) SG ducts were vital stained with novel fluorescent probe $\mathrm{SiR}-\mathrm{COOH}$, which recognizes tracheal taenidia, and scanned with laser confocal microscope (magnif. 40x). White arrow points to the common duct. (c) Chitinous character of these taenidial structures was further confirmed by their specific fluorescent staining using Fluostain (Fluorescent Brightener 28, or Calcofluor White M2R) in paraformaldehyde-fixed preparations, and subsequently scanned with a laser confocal microscope at $405 \mathrm{~nm}$ (magnif. 40x). The white arrow points to the common duct, and white arrowheads to the left and right indicate ducts connected directly to glands. (d) Short segment of the main thoracal left dorsal tracheal tube from the same larva as in Fig. 3b was used as a control and stained with SiR-COOH to show specificity of the probe for vital chitinous taenidial structures (magnif. 40x). White arrows point to the side tracheal branch. (e) Lateral tracheal tube from the same larva as in Fig. $3 \mathrm{c}$ was used as a control and stained with Fluostain to confirm specificity of the probe for chitinous taenidial structures also in a paraformaldehyde-fixed sample (magnif. 40x). Artificial color depth coding after deconvolution and maximum intensity rendering was applied to all four of these images [(b), (c), (d), (e)] to highlight the structure of circumferential rings. Thus, yellow and red structures are closer to the surface, while green is deeper and blue deepest. (f) Salivary gland ducts stained with Alexa Fluor 488 phalloidin show that the actin signal is diffused and does not align with taenidia. The orientation of SG ducts is the same as in (b) and (c). (g) The same salivary gland ducts co-stained with Cy5-anti-fasciclin III monoclonal antibody to show borders (membranes) of individual ductal cells highlighting that taenidial ridges in SG ducts also extend beyond the limits of single cells forming a supracellular structure; for a better comparison see images (h) and (i). (h) The same salivary gland ducts co-stained with Cy3-anti-tubulin polyclonal antibody illustrating clear microtubular signal in the same fashion as taenidial circumferential rings. (i) The same salivary gland ducts co-stained with Fluostain (FB28) to detect specifically chitin in the taenidia. All images were taken at magnif. 40× (oil objective lens), and scale bar $=20 \mu \mathrm{m}$ applies for all.

fusion protein in salivary gland cells. To obtain the most reliable data, we collected a series of at least $50 \mathrm{Z}$-stack optical sections (300 $\mathrm{nm}$ each); after deconvolution we performed 3D reconstruction rendering using transparency mode of Zeiss AxioVision software. As shown in Fig. $5 \mathrm{~g}$ and $5 \mathrm{~h}$, neither the expression of Sgs3-Gal4 nor dri-Gal4 led to any change in the Actin-5C-GFP signal for the SG ducts of late third-instar larvae. Due to certain properties of Sgs3-Gal4 and dri-Gal4 discussed below, we decided to also use a $h s$-Gal4 construct to drive the expression of $U A S$-Act5C-GFP ubiquitously. This was achieved by performing a $1-\mathrm{h}$ heat shock of $30^{\circ} \mathrm{C}$ at the early wandering stage of third-instar larvae, and checking for a GFP signal $2 \mathrm{~h}$ later. Under this condition we were able to detect a faint F-actin signal in the SG duct, which resembled bundles of filaments parallel to taenidial rings (Fig. 5i). However, it 

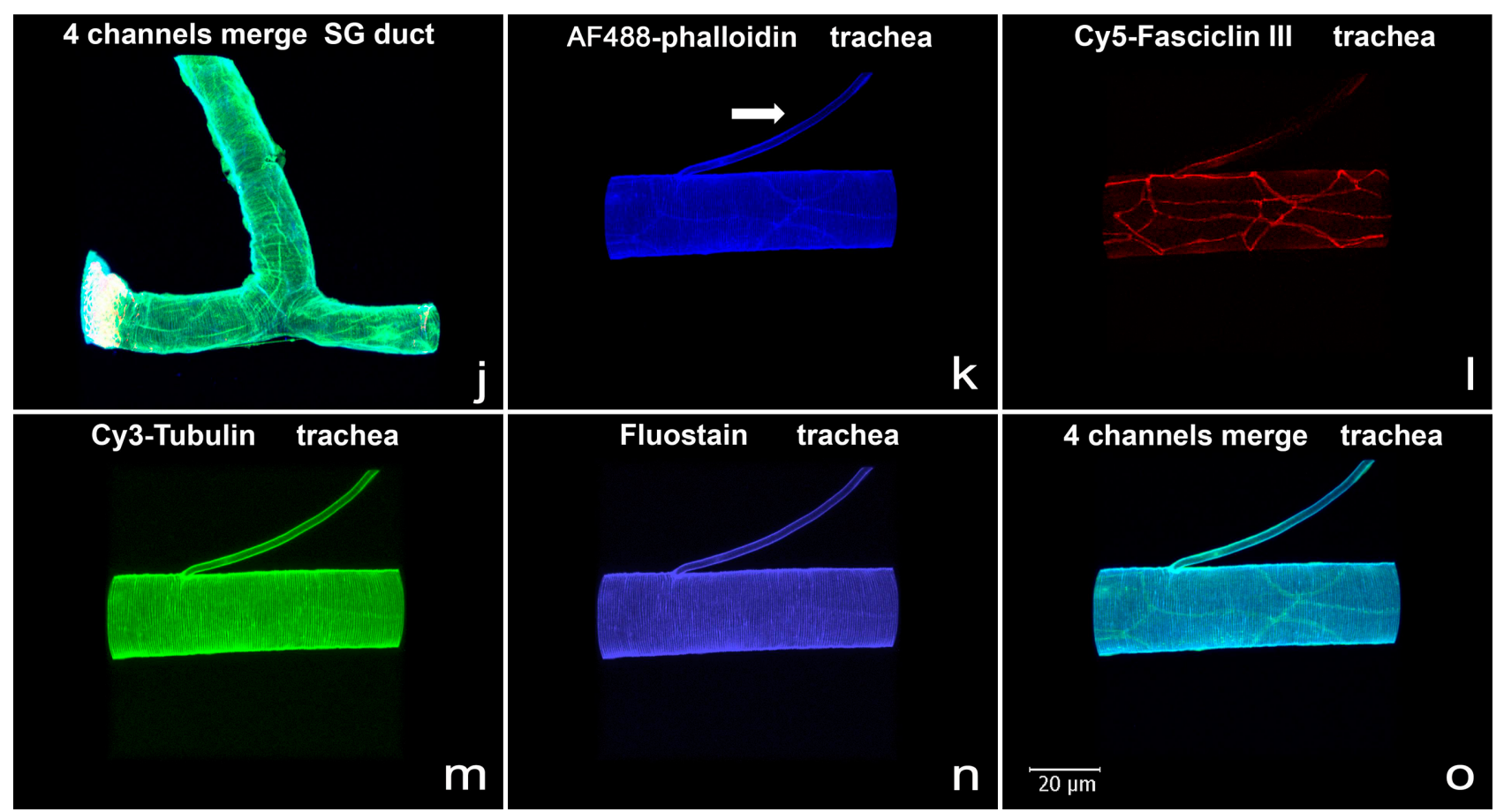

Fig. 3j-o. Salivary gland ducts (j) and trachea $(k-0)$ of Drosophila larvae. (j) This image represents the merge of the previous four channels for filamentous actin, microtubules, fasciclin III and chitin in the SG duct. (k) Main visceral left dorsal tracheal trunk from the abdomen of the same larva as in Figs $3 \mathrm{f}$ to $\mathrm{j}$, stained with Alexa Fluor 488 phalloidin to show that the signal of filamentous actin strongly underlines taenidial circumferential rings although, as seen in the merge (o), it does not necessarily colocalize. (I) The same tracheal trunk co-stained with Cy5-anti-fasciclin III monoclonal antibody to show borders (membranes) of individual ductal cells. $(\mathrm{m})$ The same tracheal trunk co-stained with Cy3-anti-tubulin polyclonal antibody showing a strong microtubular signal organized in the same fashion as taenidial circumferential rings. (n) The same tracheal trunk co-stained with Fluostain (FB28) to specifically detect chitin in the taenidia. (o) Merge of the previous four channels for filamentous actin, microtubules, fasciclin III and chitin in the tracheal trunk. All images were taken at magnif. 40× (oil objective lens), and scale bar $=20 \mu \mathrm{m}$ applies for all.

should be noted that the structures shown in Fig. $5 \mathrm{i}$ became visible only after deconvolution and $3 \mathrm{D}$ rendering.

\section{DISCUSSION}

Since their first mention by Robertson (1936) and slightly more detailed description by Ross in 1939 and her camera obscura drawings of the SG duct, there were very few references to or documentation of the circumferential chitinous lining (taenidia) in larval SGs of Drosophila melanogaster for decades. In fact, the presence of duct taenidia was not documented until the late 1970s by Berendes \& Ashburner (1978) who used light microscopy on the SGs of larval D. hydei. Several decades later, Haberman et al. (2003) studied the embryonic specification of cell fates within the salivary gland primordium, including salivary ducts, where they observed the formation of taenidia-like structures during the embryogenesis of $D$. melanogaster. The existence of taenidia inside the ducts of adult Drosophila salivary glands was only noticed by Miller (1950), although adult glands were never the subject of such intense studies as was the case for the larval organ. Furthermore, adult SGs are a completely different organ to larval glands whose polytene cells histolyse during metamorphosis. The taenidia are distinctive of the tracheal system, and are defined as circumferential annular thickenings of the cuticle inside a trachea or tracheole where they show various geometries such as loops or helices (Snodgrass, 1935; Richards, 1951; Eidmann \&
Kühlhorn, 1970; Wigglesworth, 1972; Mill, 1972, 1998; Noirot \& Noirot-Timothee, 1982; Manning \& Krasnow, 1993). From our confocal and SEM observations it is apparent that larval SGs of Drosophila contain highly similar, if not identically composed taenidial structures inside the ductal tubes, and most probably serve as the primary source of its scaffolding structural integrity and reinforcement as in trachea.

Some of the mechanical properties of ducts, and notably taenidia, could be more appropriately assessed if we know, for example, the composition and distribution of resilin and sclerotin. Resilin, in particular, is known to be responsible for the deformability and flexibility in the membrane and joint systems of insects (Michels, 2007) and could therefore provide valuable information. Unfortunately, resilin antibody (Burrows et al., 2011; Wong et al., 2012) has not been available for at least the last four to five years, and sclerotin is a biochemically variable multiprotein complex against which no antibody can be generated since it incorporates different proteins in varying proportions in different body structures. We considered the autofluorescent technique described by Zill et al. (2000) to detect resilin or sclerotin, which has been subsequently applied to insects and some other arthropod exoskeletons (Klaus et al., 2003; Schawaroch et al., 2005; Klaus \& Schawaroch, 2006; Michels \& Gorb, 2012; Klaus et al., 2014); however, despite our repeated trials we found that this does not 

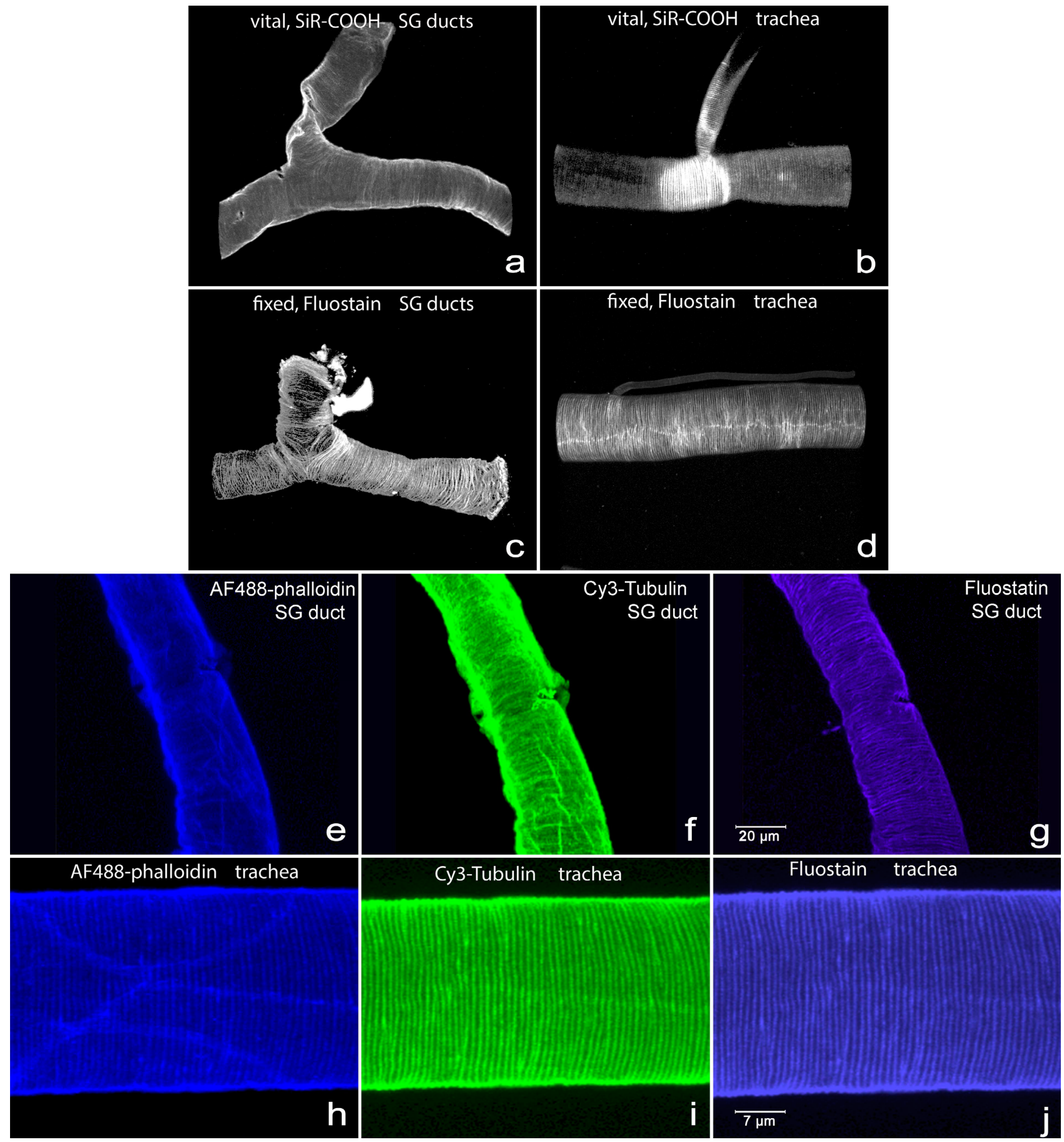

Fig. 4. Monochromatic and detailed (large-scale) presentation of salivary gland ducts and tracheae of Drosophila larvae. (a) Monochromatic presentation of the vital staining of the SG ducts with novel fluorescent probe SiR-COOH. (b) Monochromatic presentation of the vital staining of a segment of the main thoracal left dorsal tracheal tube from the same larva as in Fig. 3d with SiR-COOH. (c) Monochromatic presentation of taenidial structures in SG duct detected specifically by Fluostain (FB28) in a paraformaldehyde-fixed sample. (d) Monochromatic view of the segment of the main thoracal left dorsal tracheal tube stained by Fluostain (FB28) in a paraformaldehyde-fixed sample. (e) Detailed view of diffused signal of the filamentous actin in SG duct detected using Alexa Fluor 488 phalloidin. (f) Detailed view of apparent taenidial ridges in the same SG duct detected by anti- $\beta$-Tubulin polyclonal antibody. (g) Detailed view of clear taenidia inside SG duct as detected by chitin-specific probe Fluostain (FB28). (h) Detailed view of the pattern of filamentous actin in main dorsal left tracheal trunk corresponding with the arrangement of taenidial circumferential rings. (i) Detailed view of the pattern of microtubules in main dorsal left tracheal trunk, detected by anti- $\beta$-Tubulin polyclonal antibody, and highlighting circumferential rings of taenidia. (j) Detailed view of tracheal taenidia detected directly as chitin recognized by the Fluostain (FB28) probe. Scale bar $=20 \mu \mathrm{m}$ for (a) to (g), and scale bar $=7 \mu \mathrm{m}$ for (h) to (j).

work for ductal structures. This can be explained not only by the much softer nature of the SG ducts, which contain less resilin and sclerotin than the extremely hard and thick exoskeleton of adult beetles, crickets and grasshoppers, but mainly by the fact that sclerotin protein composition can be unique to these exoskeletal structures, which allows for bright or at least usable autofluorescence. 

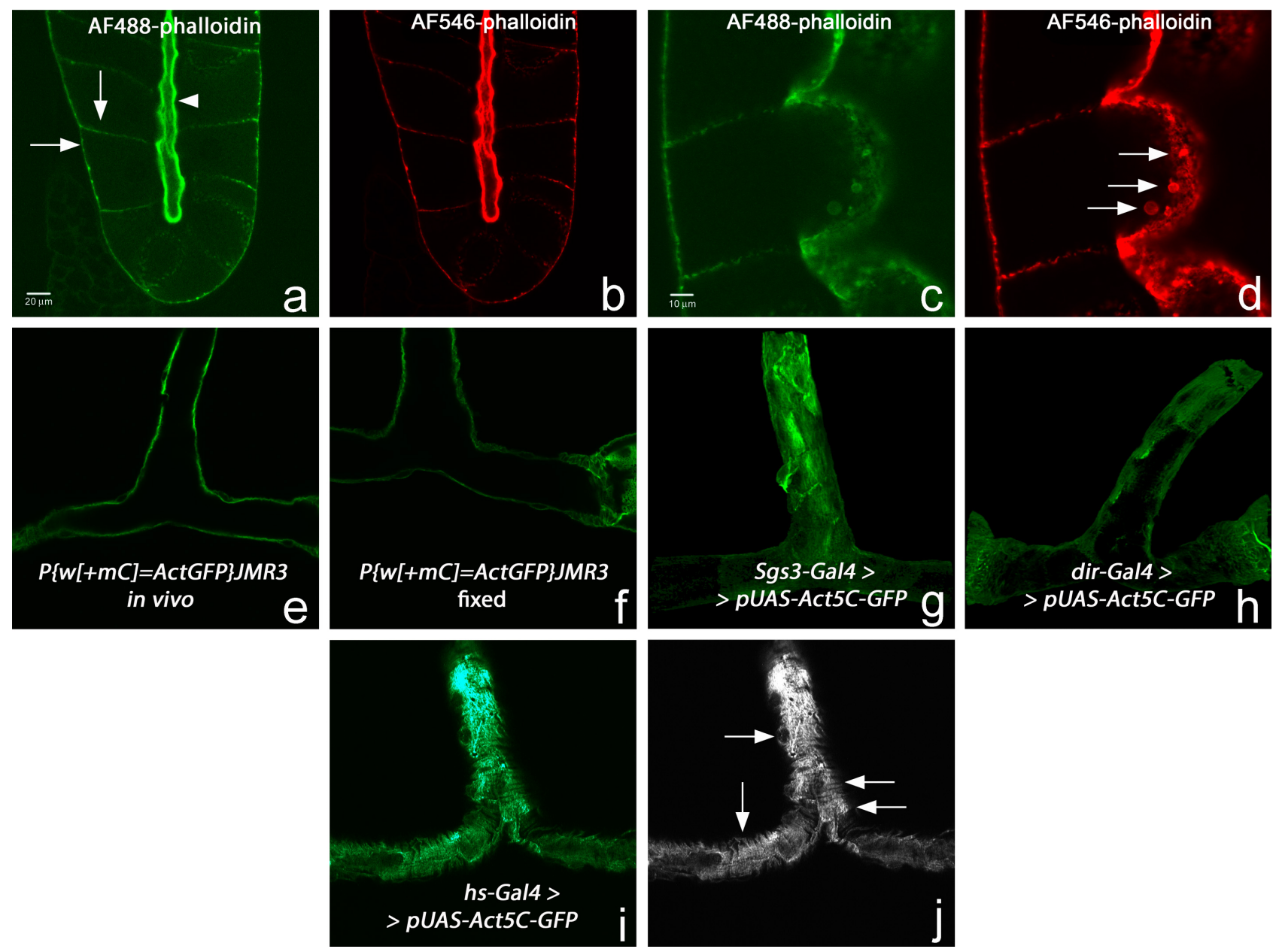

Fig. 5. Filamentous actin distribution in larval salivary glands and SG ducts of Drosophila. (a) Alexa Fluor 488 phalloidin and (b) Alexa Fluor 546 phalloidin detection of cortical actin in pre-wandering third-instar larval SGs. Arrows point to typical membrane-associated cortical locations, and the white arrowhead indicates a strong signal of filamentous actin on the apical membrane lining of the SG lumen. (c) Alexa Fluor 488 phalloidin and (d) Alexa Fluor 546 phalloidin detection of secretory vesicles in wandering stage third-instar larvae during active exocytosis of Sgs-glue granules into the lumen. Process of vesicle docking and priming to apical membrane prior to exocytotic event is associated with a strong F-actin signal. White arrows point to docked secretory granules. (e) Actin-GFP signal in living SG ducts mounted in PBS with $20 \%$ glycerol under coverslip, and (f) actin-GFP signal in paraformaldehyde-fixed prep mounted under Elvanol. In both cases the signal is relatively weak and does not show structures of the circumferential ring lining. (g) GFP signal of UAS-Act5C-GFP transgenic construct driven by Sgs3-Gal4, while (h) is the same construct expressed under the control of dri-Gal4. (i) GFP signal of UAS-Act5C-GFP transgenic construct induced temporarily by a short heat shock $\left(30^{\circ} \mathrm{C}\right)$ via $h s-G a l 4$ driver where a faint signal of F-actin in the SG duct resembling bundles of filaments parallel to taenidial rings can be seen. (j) Grayscale image of (i) to provide better visibility of the ring bundles of F-actin indicated by white arrows on the both individual and common ducts. Scale bar in (c) and (d) $=10 \mu \mathrm{m}$, scale bar elsewhere $=20 \mu \mathrm{m}$.

Although the fluorescent signal emitted from the binding of SiR-COOH or Fluostain to ductal taenidia is weaker than the signal from tracheal tubules, meaning that they may contain less chitin, these ductal taenidia can provide sufficient strength and flexibility to the duct. Importantly, the taenidia may prevent collapse of the ductal tubule during ecdysis in a similar way as for tracheae (Matusek et al., 2006; Hannezo et al., 2015), at least during the larval stages. The common duct of the SGs opens in the floor of the muscular pharynx, the portion of anterior armature chiefly involved in foraging (Sokolowski et al., 1997), which is the permanent locomotor activity of the larval foregut since hatching of the first-instar larva until the prepupa. This continuous muscular activity requires highly elastic connections that are able to resist mechanical tension; duct taenidia meet this specification. Moreover, there is a good reason to believe that taenidia also prevent collapse of the duct during glue expectoration. This is a time when the larva undergoes very strong extracardiac pulsations executed by the abdominal musculature, generating high internal hemolymph pressure to facilitate body contractions at the onset of pupariation (Žd'árek \& Fraenkel, 1972; Žd'árek, 1985). These hemolymph pressure pulsations in Sarcophaga flies and some other holometabolic insects reach 100 to $300 \mathrm{~mm} \mathrm{Hg}$ ( 10 to $40 \mathrm{kPa}$ ) of internal pressure during pupariation (Sláma, 1976, 1984, 2000; Žd'árek et al., 1979; Žd'árek, 1985). We estimate that in the much smaller Drosophila, the immobilization and contraction phase of the pupariation would result in 2 to $5 \mathrm{kPa}$ of internal hemolymph pressure, still significantly above atmospheric level. Many internal organs, including salivary glands and their duct, must therefore posses the ability to resist this distort- 
ing influence and to return to their original size and shape after the repeated contraction cycles or waves. In contrast to the rest of the salivary gland where corpuscular, transitional, and columnar secretory cells are relatively thick and volumetric, ductal cells are very thin and narrow. Thus the "corrugated hose of a vacuum cleaner" (a term originally used to describe tracheae by Manning \& Krasnow, 1993) can serve this mechanical purpose very well. An additional key property of duct taenidia could therefore be the ability to recoil from compression during the secretory cycle.

Having provided structural and histochemical information on the identity of taenidia inside SG ducts, the challenge now is to analyze their development and formation using the series of genetic tools available for Drosophila. In the SGs of late third instar Drosophila larvae we were unable to obtain a clear pattern of the filamentous actin rings that corkscrew around the lumen of the ducts and line or colocalize with the taenidia as described for embryonic SG ducts by Haberman et al. (2003). It has been clearly established in Drosophila trachaea that F-actin bundle organization is important for embryonic taenidium formation (Chihara et al., 2003; Matusek et al., 2006); the situation in SG ducts may be very similar. Our observations indicate that this early morphogenetic function of F-actin in SG duct tubules may be limited to prefiguring the cuticular ridges during embryogenesis. There are at least two plausible explanations for this. First, actin that is primarily required for prefiguring the cuticular ridges in embryos is subsequently diluted during postembryonic development by simple mechanical expansion of the ductal tube. The tube grows only by volumetric expansion, gradually making filamentous bundles less visible, although they still may persist from the embryonic period. It should be emphasized that the most rapid expansion of the SG duct takes place during the second half of the late third instar, when the SG proper grows significantly due to the huge production of Sgs-secretory granules. At this point, the SGs increase in size more than 20 -fold. This could explain why much-less diluted F-actin bundles are detectable in second-instar larvae, as observed by Haberman et al. (2003). Secondly, during larval development and towards metamorphosis, the originally embryonic prefiguring function of F-actin is no longer required and is exchanged or replaced by microtubular scaffold. This is easy to detect by both anti-tubulin specific antibodies and by fluorescently-conjugated microtubule-binding probes (colchicine or paclitaxel). In fact, it is plausible that both of the abovementioned explanations are true and are complementary. The absence of F-actin bundles in third-instar larval SG ducts is in striking contrast to that of third-instar larval tracheae where the optical pattern of actin microfilaments corresponds to the taenidial chitinous ridge pattern or is closely co-located. It needs to be stressed that both actin and tubulin are localized intracellularly, whereas chitinous ridge taenidia are an extracellular matrix (cuticle) forming a supra-cellular structure.

Experiments with actin-GFP fusion constructs have shown that expression, either under the control of an endogenous promoter or ectopic drivers (Sgs3-Gal4 or $d r i-$
Gal4) does not enhance the detection of ductal F-actin bundles in late third-instar larvae. Despite the fact the Sgs3-Gal4 driver is very strong and specific to the second half of the third instar, its expression appears to be restricted only to SG proper cells and is absent from ductal cells. The dri-Gal4 driver has not been used in similar experiments to our knowledge and is derived from the dead-ringer (also known as retained) promoter. Strong expression of the dri gene is required for determining SG ducts during Drosophila embryogenesis (Bradley et al., 2001; Vining et al., 2005; Kerman et al., 2006), and it is not known if its duct-specific expression is also maintained during postembryonic development. It may be the case that after the determination and full differentiation of embryonic SG ducts is complete, expression of $d r i$ is downregulated. Interestingly, ectopic overexpression of actin-GFP under the control of a $h s$-Gal4 driver slightly increased the detectability of circumferential rings of F-actin in the duct cells. The heat shock promoter in the $h s$-Gal4 construct is most inducible at $37^{\circ} \mathrm{C}$; however, we decided to use a lower temperature $\left(30^{\circ} \mathrm{C}\right)$ and for a limited time $(60 \mathrm{~min})$ to keep ectopic overexpression at modest levels. Despite this, it was still sufficient to produce enough ectopic actin-GFP protein to make it visible in ductal cells at locations observed during embryogenesis and early instars. This result provides strong support to our above mentioned conclusion that F-actin in the ductal cells of late third-instar larvae could become diluted during postembryonic development by mechanical expansion of the ductal tube. However, its modest ectopic overexpression can restore it to locations typical of much earlier stages. We purposely did not use a stronger and longer heat shock to avoid ectopic mislocalization of F-actin in inappropriate subcellular locations.

Several genes that are involved in determining tubulogenesis during embryonic development are identical for both the tracheal system and for SGs (Kuo et al., 1996; Henderson \& Andrew, 2000; Jin et al., 2001; Kerman et al., 2006). Based on our observations, one can anticipate that at least some of the genes which affect taenidial formation in a tracheal system (Öztürk-Çolak et al., 2016) may be different from the genes required to determine and maintain taenidia in SG ducts. In addition, the presented observations may indicate a crucial difference between the fate of SGs and the tracheal system: SGs (including their ducts) are ultimately doomed for histolysis, whereas the tracheal system remains functional during metamorphosis, and undergoes extensive remodeling (Whitten, 1980; Manning \& Krasnow, 1993; Weaver \& Krasnow, 2008; Pitsouli \& Perrimon, 2010; Hayashi \& Kondo, 2018). The different distribution of F-actin can be an indicator forecasting this fate.

ACKNOWLEDGEMENTS. We acknowledge K. Sláma's advice and helpful discussions on hemolymph pressure pulsations. We are indebted to G. Lukinavičius and S.W. Hell of the Max Planck Institute for Biophysical Chemistry (Göttingen, Germany) for their generous gift of novel fluorescent probes SiR$\mathrm{COOH}$ and 580CP-LTX. We also thank A.J. Andres (University of Nevada, Las Vegas, NV, USA) and Bloomington Drosophila Stock Center (Indiana University, Bloomington, IN, USA) for fly 
stocks, and DSHB (Iowa University, Iowa City, IA, USA) for the anti-fasciclin-III monoclonal antibody. This work was supported by VEGA 2/0109/13 and VEGA 2/0103/17, by NATO grants CRG-972173 and LST.CLG-977559, by MVTS-32060600/ECInstruct-FP7-211252 grant, by COST ENBA-CA15216 grant, EEA-Norwegian FM SK-0086 grant, and APVV-16-0219 grant to R.F. The excellent technical assistance of M. Bardačová is also acknowledged.

\section{REFERENCES}

Abrams E.W., Vining M.S. \& AndRew D.J. 2003: Constructing an organ: the Drosophila salivary gland as a model for tube formation. - Trends Cell Biol. 13: 247-254.

Ashburner M. 1970: The genetic analysis of puffing in polytene chromosomes of Drosophila. — Proc. R. Soc. Lond. (B) $\mathbf{1 7 6}$ 319-327.

Ashburner M. \& Berendes H.D. 1978: Puffing of polytene chromosomes. In Ashburner M. \& Wright T.R.F. (eds): The Genetics and Biology of Drosophila. Vol. 2b. Academic Press, London, New York, pp. 315-395.

Ashburner M. \& Thompson J.N. 1978: The laboratory culture of Drosophila. In Ashburner M. \& Wright T.R.F. (eds): The Genetics and Biology of Drosophila. Vol. 2a. Academic Press, London, New York, pp. 1-109.

Beño M., Liszeková D. \& Farkaš R. 2007: Processing of soft pupae and uneclosed pharate adults of Drosophila for scanning electron microscopy. - Microsc. Res. Tech. 70: 1022-1027.

BeŇová-Liszeková D., BeŇo M. \& Farkaš R. 2019a: A protocol for processing the delicate larval and prepupal salivary glands of Drosophila for scanning electron microscopy. - Microsc. Res. Tech. 82: 1145-1156.

BeñovÁ-Liszeková D., BeŇo M. \& Farkaš R. 2019b: Fine infrastructure of released and solidified Drosophila larval salivary secretory glue using SEM. - Bioinspir. Biomim. 14: 055002, $11 \mathrm{pp}$.

BerENDES H.D. 1965: Salivary gland function and chromosomal puffing patterns in Drosophila hydei. - Chromosoma 17: $35-77$.

Berendes H.D. \& Ashburner M. 1978: The salivary glands. In Ashburner M. \& Wright T.R.F. (eds): The Genetics and Biology of Drosophila. Vol. 2a. Academic Press, London, New York, pp. 453-498.

Bodenstein D. 1950: The postembryonic development of Drosophila. In Demerec M. (ed.): Biology of Drosophila. Wiley and Sons, New York, pp. 1-35.

Bradley P.L., Haberman A.S. \& Andrew D.J. 2001: Organ formation in Drosophila: specification and morphogenesis of the salivary gland. - Bioessays 23: 901-911.

BRIDGES C.B. 1935: Salivary chromosome maps with a key to the banding of the chromosomes of Drosophila melanogaster. J. Hered. 26: 60-64.

Burrows M., Borycz J.A., Shaw S.R., Elvin C.M. \& MeinERTZHAGEN I.A. 2011: Antibody labelling of resilin in energy stores for jumping in plant sucking insects. - PLOS ONE 6: e28456, 7 pp.

Chinara T., Kato K., Taniguchi M., Ng J. \& Hayashi S. 2003: Rac promotes epithelial cell rearrangement during tracheal tubulogenesis in Drosophila. — Development 130: 1419-1428.

Da Lage J.-L., Thomas G.W.C., Bonneau M. \& Courtier-OrGOGOZo V. 2019: Evolution of salivary glue genes in Drosophila species. - BMC Evol. Biol. 19: 36, 22 pp.

EIDMANN H.A. \& KÜHLHORN F. 1970: Lehrbuch der Entomologie. 2nd ed. Paul Parey, Hamburg, Berlin, 633 pp.
FARKAŠ R. 2016: The complex secretions of the salivary glands of Drosophila melanogaster, a model system. In Cohen E. \& Moussian B. (eds): Extracellular Composite Matrices in Arthropods. Springer International Publishing, Cham, pp. 557599.

FARKAŠ R. \& ŠUŤÁKOVÁ G. 1998: Ultrastructural changes of Drosophila larval and prepupal salivary glands cultured in vitro with ecdysone. - In Vitro Cell. Dev. Biol. Anim. 34: 813-823.

Farkaš R., BeŇová-Liszeková D., Mentelová L., Mahmood S., Ďatková Z., Beňo M., Pečeňová L., RašKa O., Šmigová J., Chase B.A. et AL. 2015: Vacuole dynamics in the salivary glands of Drosophila melanogaster during prepupal development. - Dev. Growth Differ. 57: 74-96.

Farkaš R., Beñová-Liszeková D., Mentelová L., Beño M., Babišová K., Trusinová-PeČeñová L., RašKa O., Chase B.A. \& RAŠKA I. 2018: Endosomal vacuoles of the prepupal salivary glands of Drosophila play an essential role in the metabolic reallocation of iron. - Dev. Growth Differ. 60: 411-430.

Fraenkel G. \& Brookes V.J. 1953: The process by which the puparia of many species of flies become fixed to a substrate. Biol. Bull. 105: 442-449.

Haberman A.S., Isaac D.D. \& Andrew D.J. 2003: Specification of cell fates within the salivary gland primordium. - Dev. Biol. 258: 443-453.

Hannezo E., Dong B., Recho P., Joanny J.F. \& Hayashi S. 2015: Cortical instability drives periodic supracellular actin pattern formation in epithelial tubes. - Proc. Natl. Acad. Sci. U.S.A. 112: $8620-8625$.

HAYASH S. \& Kondo T. 2018: Development and function of the Drosophila tracheal system. - Genetics 209: 367-380.

Henderson K.D. \& ANDREw D.J. 2000: Regulation and function of Scr, exd, and hth in the Drosophila salivary gland. - Dev. Biol. 217: 362-374.

Jin J., Anthopoulos N., Wetsch B., Binari R.C., IsaAC D.D., AndRew D.J., Woodgett J.R. \& Manoukian A.S. 2001: Regulation of Drosophila tracheal system development by protein kinase B. - Devel. Cell 1: 817-827.

Jones N.A., Kuo Y.M., Sun Y.H. \& BeCKENDORF S.K. 1998: The Drosophila pax gene eye gone is required for embryonic salivary duct development. - Development 125: 4163-4174.

Kerman B.E., Cheshire A.M. \& Andrew D.J. 2006: From fate to function: The Drosophila trachea and salivary gland as models for tubulogenesis. - Differentiation 74: 326-348.

Klaus A.V., Kulasekera V.L. \& Schawaroch V. 2003: Threedimensional visualization of insect morphology using confocal laser scanning microscopy. - J. Microsc. 212: 107-121.

Klaus A.V. \& Schawaroch V. 2006: Novel methodology utilizing confocal laser scanning microscopy for systematic analysis in arthropods (Insecta). - Integr. Comp. Biol. 46: 207-214.

Klaus A.V., Schawaroch V. \& Frischmann K.J. 2014: Confocal imaging and three-dimensional visualization of thick autofluorescent specimens. - Methods Mol. Biol. 1075: 213-225.

KolesniKov N.N. \& ZhimULEv I.F. 1975: Synthesis of mucoprotein secretory substance in salivary glands of the Drosophila melanogaster 3rd instar larvae. - Ontogenez 6: 171-182 [in Russian].

Korge G. 1975: Chromosome puff activity and protein synthesis in larval salivary glands of Drosophila melanogaster. - Proc. Natl. Acad. Sci. U.S.A. 72: 4550-4554.

KoRge G. 1977a: Larval saliva in Drosophila melanogaster: production, composition and relationship to chromosome puffs. Dev. Biol. 58: 339-355.

Korge G. 1977b: Direct correlation between a chromosome puff and the synthesis of a larval saliva protein in Drosophila melanogaster. - Chromosoma 62: 155-174. 
Kuo Y.M., Jones N., Zhou B., Panzer S., Larson V. \& BeckenDORF S.K. 1996: Salivary duct determination in Drosophila: roles of the EGF receptor signaling pathway and the transcription factors Fork head and Trachealess. - Development 122: 1909-1917.

Lane N.J., Carter Y.R. \& Ashburner M. 1972: Puffs and salivary gland function: the fine structure of the larval and prepupal salivary glands of Drosophila melanogaster. - Wilhelm Roux Arch. 169: 216-238.

LefeVre G. 1976: A photographic representation and interpretation of the polytene chromosomes of Drosophila melanogaster salivary glands. In Ashburner M. \& Novitski E. (eds): The Genetics and Biology of Drosophila. Academic Press, London, New York, pp. 31-66.

Lehmann M. 1996: Drosophila Sgs genes: stage and tissue specificity of hormone responsivness. - BioEssays 18: 47-54.

Lindsley D.L. \& Zimm G.G. 1992: The Genome of Drosophila melanogaster. Academic Press, San Diego, CA, 1133 pp.

Lukinavičius G., Mitronova G.Y., Schnorrenberg S., Butkevich A.N., Barthel H., Belov V.N. \& Hell S.W. 2018: Fluorescent dyes and probes for super-resolution microscopy of microtubules and tracheoles in living cells and tissues. - Chem. Sci. 9: 3324-3334.

Manning G. \& Krasnow M.A. 1993: Development of the Drosophila tracheal system. In Bate M. \& Martinez Arias A. (eds) The Development of Drosophila melanogaster. Cold Spring Harbor Press, New York, pp. 609-685.

Matusek T., Ditane A., Jankovics F., Brunner D., Mlodzik M. \& MinÁLY J. 2006: The Drosophila formin DAAM regulates the tracheal cuticle pattern through organizing the actin cytoskeleton. - Development 133: 957-966.

Michels J. 2007: Confocal laser scanning microscopy: using cuticular autofluorescence for high resolution morphological imaging in small crustaceans. - J. Microsc. 227: 1-7.

Michels J. \& Gorb S.N. 2012: Detailed three-dimensional visualization of resilin in the exoskeleton of arthropods using confocal laser scanning microscopy. - J. Microsc. 245: 1-16.

Mill P.J. 1972: Respiration in the Invertebrates. MacMillan, London, Basingstoke, New York, Melbourne, $232 \mathrm{pp}$.

MiLl P.J. 1998: Tracheae and tracheoles. In Harrison F. \& Locke M. (eds): Microscopic Anatomy of Invertebrates. Vol. $11 \mathrm{~A}$ Insecta. Wiley-Liss, New York, pp. 303-336.

Miller A. 1950: The internal anatomy and histology of the imago of Drosophila melanogaster. In Demerec M. (ed.): Biology of Drosophila. Wiley \& Sons, New York, pp. 420-534.

Noirot C. \& Noirot-Timothee C. 1982: The structure and development of the tracheal system. In King R.C. \& Akai H. (eds) Insect Ultrastructure. Vol. 1. Plenum Press, New York, pp. 351-381.

Öztürk-Çolak A., Moussian B. \& AraúJo S.J. 2016: Drosophila chitinous aECM and its cellular interactions during tracheal development. - Dev. Dynam. 245: 259-267.

PAINTER T.S. 1933: A new method for the study of chromosome rearrangements and the plotting of chromosome maps. - Science 178: 585-586.

Pelling C. 1966: A replicative and synthetic chromosomal unitthe modern concept of the chromomere. - Proc. R. Soc. Lond. (B) 164: 279-289.

Pitsouli C. \& Perrimon N. 2010: Embryonic multipotent progenitors remodel the Drosophila airways during metamorphosis. - Development 137: 3615-3624.

Ransom R. 1982: A Handbook of Drosophila Development. Elsevier Biomedical Press, Amsterdam, New York, 289 pp.

Richards A.G. 1951: The Integument of Arthropods. University of Minnesota Press, Minneapolis, 428 pp.
Robertson C.W. 1936: The metamorphosis of Drosophila melanogaster, including an accurately timed account of the principal morphological changes. - J. Morphol. 59: 351-399.

Ross E.B. 1939: The post-embryonic development of the salivary gland by Drosophila melanogaster. - J. Morphol 65: 471-496.

Schawaroch V., Grimaldi D. \& Klaus A.V. 2005: Focusing on morphology: applications and implications of confocal laser scanning microscopy (Diptera: Campichoetidae, Camillidae, Drosophilidae). - Proc. Entomol. Soc. Wash. 10: 323-335.

SLÁmA K. 1976: Insect haemolymph pressure and its determination. - Acta Entomol. Bohemoslov. 73: 65-75.

SLÁmA K. 1984: Recording of haemolymph pressure pulsations from the insect body surface. - J. Comp. Physiol. (B) 154: 635-643.

Sláma K. 2000: Extracardiac versus cardiac haemocoelic pulsations in pupae of the mealworm (Tenebrio molitor L.). $-J$. Insect Physiol. 46: 977-992.

Snodgrass R.E. 1935: Principles of Insect Morphology. McGraw-Hill, New York, 667 pp.

Sokolowski M.B., Pereira H.S. \& Hughes K. 1997: Evolution of foraging behavior in Drosophila by density-dependent selection. - Proc. Natl. Acad. Sci. U.S.A. 94: 7373-7377.

SoRSA V. 1989: Chromosome Maps of Drosophila melanogaster. CRC Press, Boca Raton, FL, 257 pp.

Vining M.S., Bradley P.L., Comeaux C.A. \& Andrew D.J. 2005: Organ positioning in Drosophila requires complex tissue-tissue interactions. - Dev. Biol. 287: 19-34.

von GAUdeCKer B. 1972: Der Strukturwandel der larvalen Speicheldrüse von Drosophila melanogaster. Ein Beitrag zur Frage nach der steuernden Wirkung aktiver Gene auf das Cytoplasma. - Z. Zellforsch. Mikrosk. Anat. 127: 50-86.

Weaver M. \& Krasnow M.A. 2008: Dual origin of tissue-specific progenitor cells in Drosophila tracheal remodeling. - Science 321: 1496-1499.

Whitten J. 1980: The tracheal system. In Ashburner M. \& Wright T.R.F. (eds): The Genetics and Biology of Drosophila. Vol 2. Academic Press, New York, pp. 499-540.

Wigglesworth V.B. 1972: The Principles of Insect Physiology. 7 th $E d$. Methuen, London, 827 pp.

Wong D.C.C., Pearson R.D., Elvin C.M \& Merritt D.J. 2012: Expression of the rubber-like protein, resilin, in developing and functional insect cuticle determined using a Drosophila antirec 1 resilin antibody. - Dev. Dynam. 241: 333-339.

YAo J.G. \& Sun Y.H. 2005: Eyg and Ey Pax proteins act by distinct transcriptional mechanisms in Drosophila development. - EMBO J. 24: 2602-2612.

Zill S., Faith Frazier S., Neff D., Quimby L., Carney M., DiCAprio R., Thuma J. \& Norton M. 2000: Three-dimensional graphic reconstruction of the insect exoskeleton through confocal imaging of endogenous fluorescence. - Microsc. Res. Tech. 48: 367-384.

Ž̇̌ÁREK J. 1985: Regulation of pupariation in flies. In Kerkut G.A. \& Gilbert L.I. (eds): Comprehensive Insect Physiology, Biochemistry, and Pharmacology. Vol. 8. Pergamon Press, Oxford, New York, pp. 301-333.

ŽĎÁREK J. \& FRAENKEL G. 1972: The mechanism of puparium formation in flies. - J. Exp. Zool. 179: 315-323.

ŽĎÁReK J., Sláma K. \& Fraenkel G. 1979: Changes in internal pressure during puparium formation in flies. - J. Exp. Zool. 207: 187-196.

Received March 27, 2020; revised and accepted March 16, 2021 Published online April 19, 2021 